\title{
Regression of target organ damage in children and adolescents with primary hypertension
}

\author{
Mieczyslaw Litwin • Anna Niemirska • Joanna Śladowska-Kozlowska • \\ Aldona Wierzbicka • Roman Janas • Zbigniew T. Wawer • Andrzej Wisniewski • \\ Janusz Feber
}

Received: 5 May 2010 /Revised: 19 July 2010 / Accepted: 20 July 2010/Published online: 21 August 2010

(C) The Author(s) 2010. This article is published with open access at Springerlink.com

\begin{abstract}
We assessed the effects of 12 months of nonpharmacological and pharmacological therapy on 24-h ambulatory blood pressure, regression of target organ damage (TOD) and metabolic abnormalities in 86 children $(14.1 \pm 2.4$ years $)$ with primary hypertension. Twenty-four hour systolic and diastolic blood pressure (BP) decreased
\end{abstract}

\section{Litwin}

Department of Research,

The Children's Memorial Health Institute,

Al. Dzieci Polskich 20,

Warsaw 04-730, Poland

M. Litwin $(\varangle) \cdot$ A. Niemirska $\cdot$ J. Śladowska-Kozlowska Department of Nephrology \& Arterial Hypertension,

The Children's Memorial Health Institute,

Al. Dzieci Polskich 20,

Warsaw 04-730, Poland

e-mail: m.litwin@czd.pl

\section{A. Wierzbicka $\cdot$ Z. T. Wawer}

Department of Biochemistry \& Experimental Medicine,

The Children's Memorial Health Institute,

Al. Dzieci Polskich 20,

Warsaw 04-730, Poland

R. Janas

Department of Radioimmunology,

The Children's Memorial Health Institute,

Al. Dzieci Polskich 20,

Warsaw 04-730, Poland

\section{A. Wisniewski}

Department of Physiology,

Jozef Pilsudski University of Physical Education,

Warsaw, Poland

\section{J. Feber}

Department of Pediatrics, Division of Nephrology,

Children's Hospital of Eastern Ontario, University of Ottawa,

Ottawa, Canada
( $130 \pm 8$ vs $126 \pm 8,73 \pm 7$ vs $70 \pm 7, p=0.0001$ and 0.004 respectively). Body mass index (BMI) did not change, but waist-to-hip $(0.85 \pm 0.07$ vs $0.83 \pm 0.05, p=0.01)$ and waistto-height ratio (WHtR; $0.49 \pm 0.07$ vs $0.48 \pm 0.05, p=0.008$ ) decreased. Left ventricular mass index (LVMi; $38.5 \pm 10.7$ vs $\left.35.2 \pm 7.5 \mathrm{~g} / \mathrm{m}^{2.7}, p=0.0001\right)$, prevalence of left ventricular hypertrophy $(46.5 \%$ vs $31.4 \%$; $p=0.0001)$, carotid intimamedia thickness (cIMT; $0.44 \pm 0.05$ vs $0.42 \pm 0.04 \mathrm{~mm}, p=$ 0.0001 ), wall cross sectional area (WCSA; $7.5 \pm 1.3$ vs $6.9 \pm$ $\left.1.2 \mathrm{~mm}^{2}, p=0.002\right)$, hsCRP $(1.1 \pm 1.0$ vs $0.7 \pm 0.7 \mathrm{mg} / 1, p=$ $0.002)$, and LDL-cholesterol ( $115 \pm 33$ vs $107 \pm 26 \mathrm{mg} / \mathrm{dl}, p=$ $0.001)$ decreased. Patients who had lowered BP had a lower cIMT at the second examination $(0.41 \pm 0.04$ vs $0.43 \pm$ $0.04 \mathrm{~mm}, p=0.04)$ and lower initial hsCRP values $(0.9 \pm 0.7$ vs $1.5 \pm 1.3 \mathrm{mg} / \mathrm{l}, p=0.04$ ) in comparison to non-responders. Regression analysis revealed that the main predictor of LVMi decrease was a decrease in abdominal fat expressed as a decrease in waist circumference (WC) $\left(R^{2}=0.280, \beta=\right.$ $0.558, p=0.005)$, for WCSA-SDS a decrease in WC $\left(R^{2}=\right.$ $0.332, \beta=0.611, p=0.009)$ and for a cIMT-SDS decrease the main predictor was a decrease in hsCRP concentrations $\left(R^{2}=\right.$ $0.137, \beta=0.412, p=0.03)$. Standard antihypertensive treatment lowered BP and led to regression of TOD in hypertensive children. Lean body mass increase and decrease in abdominal obesity correlated with TOD regression.

Keywords Primary hypertension - Target organ damage . Arteriosclerosis $\cdot$ Metabolic syndrome $\cdot$ Left ventricular hypertrophy Children

\section{Introduction}

Primary hypertension (PH) in childhood and adolescence is not a benign disease and causes significant target organ 
damage (TOD), which is already present at diagnosis $[1,2]$. Although clinically evident cardiovascular disease is rare in childhood, it is known that the extent of TOD is the main risk factor for future cardiovascular events [3]. Thus, the efficacy of antihypertensive treatment should be evaluated not only in terms of blood pressure (BP) lowering, but also in terms of regression of TOD and metabolic abnormalities. As cardiovascular events are extremely rare in childhood, it is the TOD regression or progression that can be used as an intermediate end-point in prospective studies in pediatric patients. To date, there have been only limited data on the effectiveness of antihypertensive treatment in children with $\mathrm{PH}$ and even fewer data exist on the regression of TOD [4-6].

The evaluation of the effects of antihypertensive therapy, both pharmacological and non-pharmacological, on metabolic abnormalities accompanying PH in children and adolescents is challenging. Usually, both the non-pharmacological and pharmacological therapies are used together and therefore it is difficult to separate the effects of these two interventions. Obesity or being overweight is the main intermediate phenotype of children with $\mathrm{PH}$; metabolic abnormalities are linked pathophysiologically to increased abdominal obesity and are the main determinants of TOD in hypertensive children. This is why non-pharmacological treatment based on life-style modifications is supposed to be effective for both BP lowering and normalization of metabolic abnormalities. Thus, to determine the main predictors of TOD regression we performed a prospective, observational study in children with PH who underwent 12 months of standard pharmacological and non-pharmacological therapy.

\section{Patients and methods}

The study adhered to the principles of the Declaration of Helsinki and was approved by the local Ethics Committee. Informed consent was obtained from all control subjects and hypertensive patients and their parents.

\section{Patients}

All children with newly diagnosed $\mathrm{PH}$ at our institution in the period from 2005 to 2008 were considered as study candidates. We included 86 adolescents ( 66 boys, 20 girls) aged 14.1 \pm 2.4 years (range: $5-17$ ). Exclusion criteria were: presence of any significant chronic disease (except for $\mathrm{PH}$ ) including diabetes and chronic kidney disease, any acute disease including infections in the 6 weeks preceding enrollment, and incomplete data. All measurements were performed at diagnosis of $\mathrm{PH}$ and then after 12 months of standard antihypertensive therapy, including pharmacological and non-pharmacological treatment.
Primary hypertension was diagnosed after a thorough clinical and laboratory diagnostic work-up, and exclusion of known secondary causes of hypertension. In all patients serum creatinine, urea, ions, blood gases, lipids, glucose, renal Doppler ultrasound, renal scintigraphy, and urinary catecholamines were evaluated. Normal office BP values were taken from the Updated 4th Task Force Report [7]. In all patients the diagnosis was confirmed by 24-h ambulatory blood pressure monitoring (ABPM). ABPM was performed at the initial visit and at the last follow-up after 12 months. Recordings lasting at least $20 \mathrm{~h}$ with at least $80 \%$ of valid measurements were included to the analysis. BP values were calculated from the ABPM as the mean 24-h systolic (SBP) and diastolic (DBP) blood pressure and presented as absolute values and as the systolic and diastolic blood pressure index (SBPi and DBPi respectively), calculated as the ratio of SBP or DBP to the 95th percentile for height and gender based on the pediatric ABPM reference data $[8,9]$. Patients were considered to be hypertensive if their SBPi and/or DBPi were equal to or exceeded 1.0 (i.e. BP was above the 95th percentile) at the start of the therapy. We used a recently published classification system based on ABPM to classify patients as having normal blood pressure, ambulatory hypertension, and severe ambulatory hypertension [9]. Ambulatory hypertension was defined as mean ambulatory SBP above the 95th percentile and SBP load between 25 and 50\%, and severe ambulatory hypertension was defined as mean ambulatory SBP above the 95th percentile and SBP load above $50 \%$. Normotension was defined as mean ambulatory SBP below the 95th percentile and SBP load below 25\%. Pre-hypertension was defined as the mean SBP below the 95th percentile and SBP load was in the range $25-50 \%$.

All patients underwent the following assessments: anthropometrical measurements including body mass index (BMI), waist-to-hip ratio (WHR), and waist-to-height ratio (WHtR), blood pressure, carotid and femoral superficial artery intima-media thickness (cIMT and fIMT respectively), left ventricular mass index (LVMi), urinary albumin excretion, oral glucose tolerance test (OGTT), serum insulin, blood lipids, serum homocysteine, serum uric acid, and C-reactive protein (hsCRP). Obesity and being overweight were diagnosed according to the International Obesity Task Force (IOTF) recommendations [10]

Intima-media thickness measurements

Carotid and femoral superficial artery intima-media thickness (cIMT and fIMT) were evaluated by ultrasound according to the methodology described previously [11]. The median and standard deviation (SD) of normal values for cIMT and fIMT were obtained from a study of 250 healthy children published elsewhere [11]. 
The following parameters were calculated according to formulas described previously [12]:

- Mean systolic diameter $(\mathrm{sD})=(\mathrm{LsD}+\mathrm{RsD}) / 2$, where LsD - left cca systolic diameter; and RsD -right cca systolic diameter

- Mean diastolic diameter $(\mathrm{dD})=(\mathrm{RdD}+\mathrm{LdD}) / 2$, where $\mathrm{RdD}$ - right cca diastolic diameter, andLdD - left cca diastolic diameter

- Mean wall cross sectional area $(\mathrm{WCSA})=\pi(\mathrm{dD} / 2+\mathrm{IMT})$ $2-\pi(\mathrm{dD} / 2) 2$

\section{Echocardiography}

All echocardiography (ECHO) examinations were performed by one examiner who knew the clinical diagnosis, but was not aware of the severity of $\mathrm{PH}$ and the effectiveness of treatment. ECHO measurements were performed according to American Society of Echocardiography guidelines. To standardize the left ventricular mass to height, the left ventricular mass index (LVMi) was calculated according to the deSimone formula [13, 14]. Left ventricular hypertrophy ( $\mathrm{LVH}$ ) was defined as LVMi value above $38.6 \mathrm{~g} / \mathrm{m}^{2.7}$ (95th percentile), and a LVMi equal to or greater than $51 \mathrm{~g} / \mathrm{m}^{2.7}$ was the cut-off for severe LVH [7, 12-14]. In addition, because it was recently proposed that the 95th percentile of LVMi indexed to height should also be age- and gender-specific we used reference data published by Khoury et al. [15].

\section{Laboratory investigations}

Blood samples were taken after $12 \mathrm{~h}$ of fasting and were immediately sent to the laboratory. OGTT was done after oral ingestion of $1 \mathrm{~g} / \mathrm{kg}$ (maximum $75 \mathrm{~g}$ ) of glucose. The blood samples were taken via venous catheter at 30-min intervals. The plasma glucose level was measured by a Dimension analyzer. Plasma insulin concentration was measured by radioimmunoassay (RIA). Serum adiponectin and leptin levels were measured by an ELISA using a commercially available kit (LINCO Research).

Insulin resistance was expressed as homeostasis model assessment for insulin resistance (HOMA-IR) and as the Insulin Sensitivity Index (ISI $[0,120]$ ) according to the formula developed by Gutt et al. [16]. ISI $[0,120]$ highly correlated with insulin sensitivity measured by the euglycemic hyperinsulinemic clamp method. ISI was defined as (m/MPG/logMSI) where:

- $\mathrm{m}=$ [ingested glucose in $\mathrm{mg}+$ (fasting glucose in $\mathrm{mg} / \mathrm{dl}-$ 2-h glucose in $\mathrm{mg} / \mathrm{dl}) \times 0.19 \times$ body weight $(\mathrm{kg})] / 120 \mathrm{~min}$

- $\quad \mathrm{MPG}=$ mean of fasting and 2-h glucose concentrations (in $\mathrm{mg} / \mathrm{dl}$ )
- $\quad$ MSI = mean of fasting and 2-h insulin concentrations $(\mathrm{mU} / \mathrm{ml})$

- ISI $[0,120]$ was expressed as $\mathrm{mg} \times \mathrm{L} 2 / \mathrm{mmol} \times \mathrm{mU} \times \min$

- A low value of ISI $[0,120]$ indicates greater insulin resistance

Plasma homocysteine was measured with fluorescence polarization immunoassay (IMX ${ }^{\circledR}$; Abbott) for the quantitative measurement of total L-homocysteine. The hsCRP concentration was determined using highly-sensitive immunoturbidimetry (Orion Diagnostica). Serum creatinine was measured with a modified Jaffe reaction. Glomerular filtration rate was estimated using the Schwartz formula with a $\mathrm{K}$ value of 0.55 for girls aged 13-17 and children below 13 years of age, and a $\mathrm{K}$ value of 0.7 for boys aged 13-17 years of age [17].

\section{Definition of metabolic syndrome}

Because there are no national reference data for waist circumference in children, metabolic syndrome (MS) was diagnosed according to criteria described previously [18].

\section{Antihypertensive treatment}

At enrollment no one patient was treated pharmacologically or received any advice about non-pharmacological treatment. After diagnosis of $\mathrm{PH}$ all patients were informed about the principles of non-pharmacological treatment including life-style changes, increase in physical activity, and dietary modifications including salt avoidance, and were referred to an external consultant (AW). We encouraged patients to increase general physical activity up to 90 min daily including sport activity, walking, climbing stairs, etc. The nature of the activity (playing football, jogging, aerobic, walking, biking, etc.) was chosen by patients according to personal preferences. Dietary modifications were based on a significant reduction of salt use both by patients and family members, and a significant reduction in sugar.

Pharmacological treatment was immediately started in patients who had a significant TOD (LVMi $>51 \mathrm{~g} /$ height $^{2.7}$ and/or cIMT or WCSA $>2$ SDS) and/or severe ambulatory hypertension. BP was checked at 3 and 6 months during ambulatory visits. If after 3 or 6 months office BP was still within the hypertensive range, ABPM was performed. In patients who still had ambulatory or severe ambulatory hypertension, pharmacotherapy was started or another drug was given. Finally, ABPM was performed again after 12 months of treatment.

Patients who after 12 months of treatment had lower ABP below the 95th percentile were treated as responders and those who did not have lower BP below the 95th 
percentile as non-responders. Drug therapy was based on the angiotensin-converting enzyme inhibitor (ACEi) enalapril $(0.2-0.3 \mathrm{mg} / \mathrm{kg} / \mathrm{day}$ bid). Patients who had asthma or did not tolerate ACEi were prescribed the angiotensin 2 receptor type 1 blocker (ARB) losartan at a dosage of $0.7-$ $1 \mathrm{mg} / \mathrm{kg} /$ day in one or two daily doses. If patients were unable to buy the prescribed medications (enalapril or losartan), amlodipine was used with a standard dosage of $5 \mathrm{mg}$ once daily.

All patients, both those treated only non-pharmacologically and those treated non-pharmacologically and with antihypertensive drugs were evaluated in regular 3-month intervals during routine ambulatory, out-patient visits. The adherence to lifestyle modifications was assessed by interview with patients and parents. When the antihypertensive effect was inadequate, amlodipine as the second drug was prescribed and in patients treated only with lifestyle intervention, therapy with ACEi, ARB or amlodipine was started. Patients who were still hypertensive after 6 months and fulfilled the criteria of stage 2 hypertension based on home and casual BP measurements were prescribed metoprolol at a dosage of $0.5 \mathrm{mg} /$ day as the third drug.

\section{Statistical analysis}

Because the groups analyzed included subjects of different ages and genders, BMI and IMT values were expressed both as absolute values and as SDS for age and gender. LVM values in grams were standardized to height in meters $^{2.7}$ and were also expressed as SDS for age and gender. The change in the measured parameters was expressed as a delta value $(\Delta)$, i.e. the difference between measurement at 12 months and at the start of treatment.

Homogeneity of variance was checked with Levene's test. Continuous variables with a normal distribution were compared using Student's $t$ test for independent variables. Continuous values with non-normal distribution were compared using the Mann-Whitney $U$ test. Measured parameters at the start and after 12 months of treatment were compared using the Wilcoxon test. Dichotomous variables were compared using the Chi-squared test.

Correlation analysis was performed using Spearman's test. LVMi, absolute and standardized IMT, and $\Delta$ for blood pressure, and LVMi and markers of arterial wall thickness were dependent variables. Because in correlation analysis and step-wise regression analysis the dependent variables were standardized values of LVM and IMT, both genders were analyzed together. Variables with significant correlation were then included in the step-wise multiple regression analysis.

A $p$ value less than 0.05 was regarded as statistically significant. A $p$ value ranging between 0.05 and 0.1 was regarded as a statistical tendency.

\section{Results}

Clinical and demographic characteristics

At the start of treatment, 36 out of 86 (41.9\%) patients had severe ambulatory hypertension and $50(58.1 \%)$ had ambulatory hypertension. On average, patients were obese and their mean $( \pm \mathrm{SD})$ BMI-SDS was $1.8 \pm 1.8$. Being overweight or obese according to the IOTF criteria was diagnosed in $56(65.1 \%)$ and $21(24.4 \%)$ patients respectively. Metabolic syndrome was diagnosed in 13 out of 86 (15.1\%). According to definitions used in the 4th Task Report and European Guidelines, LVH was found in 40 $(46.5 \%)$ and severe LVH was present in 10 patients $(11.6 \%$; Table 1). When we used age- and gender-specific referential values of 95th percentile of LVMI, LVH was diagnosed in 39 patients $(45.3 \%)$.

\section{Antihypertensive efficacy of treatment}

Throughout the study, 37 patients $(43 \%)$ who had ambulatory hypertension at the start and who were free of TOD were prescribed only non-pharmacological therapy, including dietary advice and an increase in physical activity. Overall, 49 patients were treated pharmacologically of whom 40 with LVH and/or severe ambulatory hypertension received antihypertensive treatment at the start. Thirty-three patients $(38.3 \%)$ received one antihypertensive medication throughout the study, in 12 patients (14\%) the second drug was added after 3 months of treatment with one drug, and in 4 patients $(4.7 \%)$ the third drug was added after 6 months of treatment (Fig. 1).

Overall, after 12 months, the blood pressure lowered below the 95th percentile in 64 patients $(74.4 \%$; responders; 19 girls, 45 boys). In 10 out of 64 patients in whom SBP lowered below the 95th percentile the SBP load was still within the range $25-50 \%$ and they fulfilled criteria to diagnose pre-hypertension. Twenty-one patients $(24.4 \%$; 1 girl, 20 boys) still had ambulatory hypertension and 1 $(1.2 \%)$ had severe ambulatory hypertension, and the decrease in the severity of hypertension was significant $\left(\chi^{2}=8.151 ; p=0.004\right)$. Patients who had normalized BP did not differ in terms of age in comparison to those patients who did not have normalized BP ( $14.0 \pm 3.0$ vs $14.7 \pm 1.5$, n.s.). BP normalized significantly more often in girls than in boys $\left(\chi^{2}=9.856 ; p=0.002\right)$.

Of the 37 patients in whom only non-pharmacological treatment was prescribed 26 patients had lowered BP and 11 were still hypertensive (ambulatory hypertension). Of 49 patients treated pharmacologically and nonpharmacologically, 38 patients had lowered BP, in 10 patients BP was still hypertensive (ambulatory hypertension), and 1 patient still had severe ambulatory hyper- 
Table 1 Descriptive

demographic, anthropometrical, and hemodynamic data obtained at the start and after 12 months of treatment
BMI body mass index; SBP systolic blood pressure; DBP diastolic blood pressure

\begin{tabular}{llll}
\hline Variable & At the start & $\begin{array}{l}\text { Second evaluation after 12 months } \\
\text { of treatment }\end{array}$ & $p$ \\
\hline Girls/boys & $20 / 66$ & & \\
Mean age (years) & $14.1 \pm 2.4$ & $172.2 \pm 13.5$ & 0.0001 \\
Height (cm) & $169.4 \pm 14.8$ & $75.8 \pm 17.4$ & 0.0001 \\
Body mass (kg) & $72.9 \pm 18.8$ & $25.3 \pm 4.3$ & n.s. \\
BMI (kg/m ${ }^{2}$ ) & $25.1 \pm 4.7$ & $1.8 \pm 1.7$ & n.s. \\
BMI-SDS & $1.8 \pm 1.8$ & $0.83 \pm 0.05$ & 0.01 \\
Waist-to-hip ratio & $0.85 \pm 0.07$ & $0.48 \pm 0.05$ & 0.008 \\
Waist-to-height ratio & $0.49 \pm 0.07$ & $54(62.8 \%)$ & 0.0001 \\
Normotension & 0 & $10(11.6 \%)$ & 0.0001 \\
Pre-hypertension & 0 & $21(24.4 \%)$ & 0.004 \\
Ambulatory hypertension & $50(58.1 \%)$ & $1(1.2 \%)$ & 0.004 \\
Severe ambulatory & $36(41.9 \%)$ & $126 \pm 8$ & 0.0001 \\
hypertension & $130 \pm 8$ & $0.99 \pm 0.06$ & 0.0001 \\
24 h SBP (mmHg) & $1.03 \pm 0.07$ & $70 \pm 7$ & 0.004 \\
24 h SBP index & $73 \pm 7$ & $0.89 \pm 0.1$ & 0.002 \\
24 h DBP (mmHg) & $0.95 \pm 0.1$ & $37.5 \pm 22.2$ & 0.001 \\
24 h DBP index & $52.7 \pm 28.2$ & & \\
24 h SBP load (\%) & & & \\
\hline
\end{tabular}

tension. The hypotensive effect was greater in patients who were prescribed pharmacotherapy compared with patients treated only with life-style modifications $(-6 \pm 10$ vs $-1 \pm$ $8 \mathrm{mmHg}, p=0.01$ for $\mathrm{SBP}$ and $-5 \pm 10$ vs $0.5 \pm 5 \mathrm{mmHg}, p=$ 0.01 for DBP).

In the whole group, BMI, BMI-SDS, prevalence of being overweight (50 patients, $58.1 \%$ ) or obese (24 patients, $27.9 \%$ ) did not change, but WHR and WHtR decreased significantly. The prevalence of metabolic syndrome decreased after 12 months by $50 \%$ (13 vs 6 patients; $\mathrm{Chi}^{2}=$ 13.358, $p=0.0001)$. In addition, the total cholesterol, LDL cholesterol, hsCRP, lipoprotein (a), and leptin concentrations decreased significantly and there was tendency toward lower uric acid (Table 2).

At the start of treatment responders had lower concentrations of homocysteine $(9.5 \pm 2.5$ vs $10.6 \pm 2.0 \mu \mathrm{mol} / \mathrm{l} ; p=$ $0.04)$ and hsCRP $(0.9 \pm 0.7$ vs $1.5 \pm 1.3 \mathrm{mg} / 1 ; p=0.04)$ and tended to have lower concentrations of uric acid $(5.3 \pm 1.2$ vs $5.8 \pm 1.3 \mathrm{mg} / \mathrm{dl} ; p=0.06)$. Responders also had lower values of fIMT-SDS at the start $(0.5 \pm 2.3$ vs $1.0 \pm 1.9 ; p=$ $0.05)$ and lower cIMT after 12 months of treatment $(0.41 \pm$ 0.03 vs $0.43 \pm 0.04 \mathrm{~mm}, p=0.04)$. Insulin resistance ( $\triangle$ HOMA-IR) decreased in responders and increased in non-responders $(-0.2 \pm 1.5$ vs $0.6 \pm 1.9 ; p=0.03)$.

\section{Regression of TOD}

After 12 months of treatment the average values of LVMi, cIMT, cIMT-SDS, WCSA, and WCSA-SDS decreased significantly (Table 2). LVMi decreased significantly in
58 patients (14 girls, 44 boys, n.s.). The proportion of patients with LVH decreased from $46.5 \%$ (40 patients) at the start of treatment to $31.4 \%$ (27 patients) after 12 months $\left(\chi^{2}=23.660, p=0.0001\right)$ as well as the proportion of patients with severe LVH (from 10 cases to 1 case; $\chi^{2}$ 7.606, $p=0.006$ ). When we used age- and gender-specific reference values of the 95 th percentile of $\mathrm{LVMi}$, the proportions of patients with LVH decreased from 39 patients to $24\left(27.9 \% ; \chi^{2}=15.362, p=0.0001\right)$.

Neither the responder nor the non-responder group differed regarding prevalence of $\mathrm{LVH}$ and severe $\mathrm{LVH}$ at the start and after 12 months.

Comparison of patients divided into groups according to the regression of TOD or the stabilization/progression of TOD revealed that there were no differences between groups in terms of change in blood pressure, age, BMI, metabolic variables and type of therapy (non-pharmacological only vs non-pharmacological and pharmacological). However, the only variables that differed between groups was the decrease in abdominal fat expressed as a decrease in WHR and the waist circumference (WC), the increase in insulin sensitivity, and the larger decrease in hsCRP in patients with regression of TOD (Table 3). Moreover, the decrease in cIMT, LVMi, and WCSA did not correlate with the change in BP, but there was significant correlation between the decrease in WCSA, WCSA-SDS, and LVMi with the decrease in abdominal fat expressed as the decrease in WHR, WHtR, and WC (Figs. 2, 3; Table 4).

To find potential predictors of TOD and BP decrease step-wise regression was used. The variables that differen- 
Fig. 1 Scheme of the study design

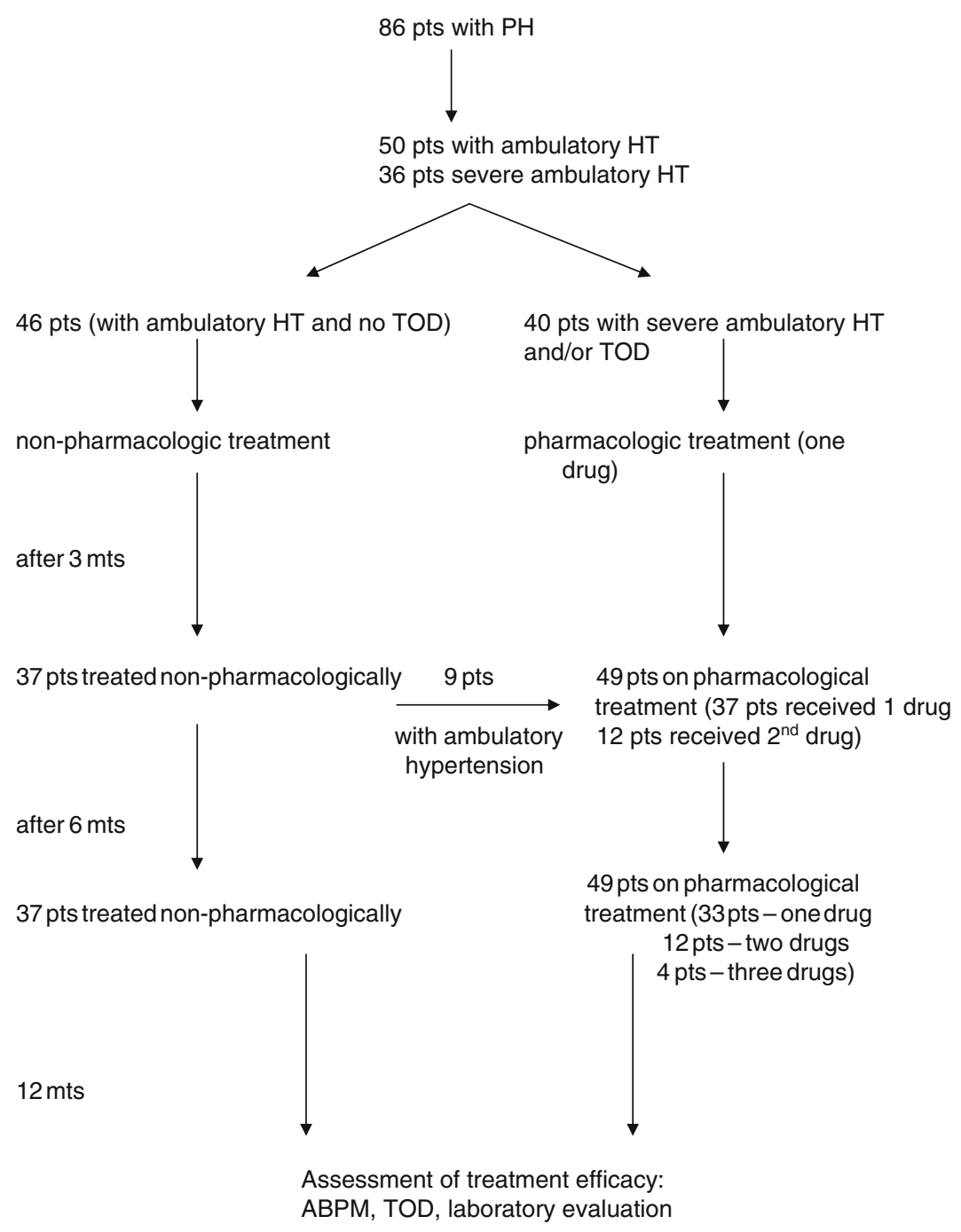

Abbreviations: ABPM - ambulatory blood pressure monitoring; $\mathrm{HT}$ - arterial hypertension; $\mathrm{PH}$ - primary hypertension; TOD - target organ damage tiated between groups of responders and non-responders, and those in whom TOD decreased or not, were chosen as independent variables. In addition, variables that correlated with TOD changes (insulin, HOMA-IR, ISI, hsCRP) were also added, as well as changes in absolute and indexed BP values. Because a decrease in visceral obesity was the main correlate of the decrease in TOD, WC was chosen as a marker of visceral fat decrease. The step-wise regression analysis revealed that the main predictor of an LVMi decrease was a decrease in abdominal fat expressed as a decrease in WC $(\beta=$ $0.558)$, explaining about $28 \%$ of variability $\left(R^{2}=0.280\right.$, $p=0.005)$, for WCSA-SDS a decrease in WC $\left(R^{2}=0.332\right.$, $\beta=0.611, p=0.009$ ), and for the cIMT-SDS decrease the main predictor was a decrease in hsCRP concentrations $\left(R^{2}=0.137, \beta=0.412, p=0.03\right)$.

\section{Discussion}

The main finding of our study is that standard nonpharmacological and pharmacological therapy for adolescents with PH lead to normalization of BP in $74 \%$ of cases, but the main determinant of regression of TOD is the decrease in abdominal obesity with an increase in lean body mass and not BP lowering.

Because diagnosis of hypertension is now confirmed by ABPM we used this method not only for confirmation of hypertension and exclusion of white coat hypertension, but also as a tool for assessment of severity of hypertension. Results of ABPM and especially of SBP load have been shown to correlate with LVMi in hypertensive children and better predicted risk of left ventricular hypertrophy than office blood pressure measurements [19-21]. However, 
Table 2 Markers of target organ damage and laboratory data obtained at the start and after 12 months of treatment

cIMT carotid intima-media thickness; WCSA carotid wall cross-sectional area; fIMT femoral intima-media thickness; HDL-cholesterol high density lipoprotein cholesterol; LDLcholesterol low density lipoprotein cholesterol; ApoA1 apoprotein A1; ApoB apoprotein B; eGFR estimated glomerular filtration rate; hsCRP high-sensitivity $\mathrm{C}$ reactive protein; HOMA-IR homeostasis model assessment for insulin resistance; ISI insulin sensitivity index

\begin{tabular}{|c|c|c|c|}
\hline Marker & At the start & After 12 months of treatment & $p$ \\
\hline cIMT (mm) & $0.44 \pm 0.05$ & $0.42 \pm 0.04$ & 0.0001 \\
\hline cIMT-SDS & $1.4 \pm 1.5$ & $0.9 \pm 1.3$ & 0.008 \\
\hline WCSA $\left(\mathrm{mm}^{2}\right)$ & $7.5 \pm 1.3$ & $6.9 \pm 1.2$ & 0.002 \\
\hline WCSA-SDS & $1.05 \pm 1.5$ & $0.2 \pm 1.3$ & 0.001 \\
\hline fIMT (mm) & $0.34 \pm 0.04$ & $0.33 \pm 0.03$ & n.s. \\
\hline fIMT-SDS & $0.01 \pm 1.4$ & $-0.2 \pm 1.1$ & n.s. \\
\hline Left ventricular mass $(\mathrm{g})$ & $161 \pm 55$ & $148 \pm 44$ & 0.0001 \\
\hline Left ventricular mass index ( $\mathrm{g} /$ height $\left.^{2.7}\right)$ & $38.5 \pm 10.7$ & $35.2 \pm 7.5$ & 0.0001 \\
\hline Left ventricular hypertrophy (\%) & $40(46.5 \%)$ & $27(31.4 \%)$ & 0.0001 \\
\hline Severe left ventricular hypertrophy (\%) & $10(11.6 \%)$ & $1(1.2 \%)$ & 0.006 \\
\hline $\begin{array}{l}\text { Left ventricular hypertrophy diagnosed } \\
\text { with age- and gender-specific cut-off values (\%) }\end{array}$ & $39(45.3 \%)$ & $24(27.9 \%)$ & 0.0001 \\
\hline Metabolic syndrome $(\%)$ & $13(15.1 \%)$ & $6(7 \%)$ & 0.0001 \\
\hline Cholesterol (mg/dl) & $175 \pm 36$ & $168 \pm 28$ & 0.02 \\
\hline Triglycerides (mg/dl) & $87 \pm 41$ & $88 \pm 38$ & n.s. \\
\hline HDL-cholesterol (mg/dl) & $44 \pm 8$ & $44 \pm 8$ & n.s. \\
\hline LDL-cholesterol (mg/dl) & $115 \pm 33$ & $107 \pm 26$ & 0.001 \\
\hline ApoA1 (mg/dl) & $1.29 \pm 0.29$ & $1.26 \pm 0.30$ & n.s. \\
\hline ApoB (mg/dl) & $0.90 \pm 0.25$ & $0.86 \pm 0.24$ & n.s. \\
\hline ApoA1/apoB & $1.5 \pm 0.4$ & $1.6 \pm 0.6$ & n.s. \\
\hline Lipoprotein (a) (mg/dl) & $18.0 \pm 14.1$ & $14.1 \pm 7.7$ & n.s. \\
\hline Uric acid (mg/dl) & $5.5 \pm 1.3$ & $5.3 \pm 1.2$ & 0.056 \\
\hline Serum creatinine $(\mathrm{mg} / \mathrm{dl})$ & $0.8 \pm 0.2$ & & \\
\hline eGFR $\left(\mathrm{ml} / \mathrm{min} / 1.73 \mathrm{~m}^{2}\right)$ & $131 \pm 18$ & & \\
\hline hsCRP (mg/l) & $1.1 \pm 1.0$ & $0.7 \pm 0.7$ & 0.002 \\
\hline Fasting glucose (mg/dl) & $84 \pm 5$ & $84 \pm 7$ & n.s. \\
\hline Fasting insulin (IU/ml) & $14.7 \pm 6.9$ & $18.2 \pm 23.1$ & n.s. \\
\hline HOMA-IR & $3.1 \pm 1.5$ & $3.2 \pm 1.6$ & n.s. \\
\hline ISI & $4.5 \pm 1.7$ & $4.7 \pm 1.7$ & n.s. \\
\hline Homocysteine $(\mu \mathrm{mol} / \mathrm{l})$ & $9.7 \pm 2.5$ & $10.0 \pm 2.6$ & n.s. \\
\hline Leptin (ng/ml) & $16.2 \pm 15.2$ & $9.8 \pm 9.5$ & 0.04 \\
\hline Adiponectin $(\mu \mathrm{g} / \mathrm{ml})$ & $10.7 \pm 4.7$ & $9.8 \pm 6.4$ & n.s. \\
\hline
\end{tabular}

Table 3 Differences between patients with regression of target organ damage (TOD) and in whom TOD was stable or progressed

\begin{tabular}{|c|c|c|c|}
\hline Variable & Decreased & Stable or increased & $p$ \\
\hline & LVMi $(n=58)$ & LVMi $(n=28)$ & \\
\hline$\Delta$ Waist-to-hip ratio & $-0.01 \pm 0.14$ & $0.07 \pm 0.02$ & 0.02 \\
\hline$\Delta$ Waist & $-3.2 \pm 6.4$ & $2.5 \pm 5.1$ & 0.001 \\
\hline \multirow[t]{2}{*}{$\triangle \mathrm{HOMA}-\mathrm{IR}$} & $-0.3 \pm 1.7$ & $0.3 \pm 1.2$ & 0.05 \\
\hline & cIMT-SDS $(n=56)$ & cIMT-SDS $(n=30)$ & \\
\hline \multirow[t]{2}{*}{$\triangle \mathrm{CRP}$} & $-0.5 \pm 1.1$ & $-0.07 \pm 0.3$ & 0.05 \\
\hline & WCSA-SDS $(n=56)$ & WCSA $(n=30)$ & \\
\hline$\Delta$ Waist-to-hip ratio & $-0.05 \pm 0.06$ & $0.02 \pm 0.03$ & 0.05 \\
\hline$\Delta$ Waist circumference & $-4.8 \pm 7.5$ & $3.0 \pm 5.3$ & 0.005 \\
\hline$\Delta \mathrm{ISI}$ & $0.6 \pm 1.4$ & $-0.5 \pm 1.2$ & 0.02 \\
\hline
\end{tabular}

Only the significant differences are shown

LVMi left ventricular mass index; HOMA-IR homeostasis model assessment for insulin resistance; ISI insulin sensitivity index; hsCRP high sensitivity $\mathrm{C}$ reactive protein; WCSA carotid wall cross-sectional area; cIMT carotid intima-media thickness 


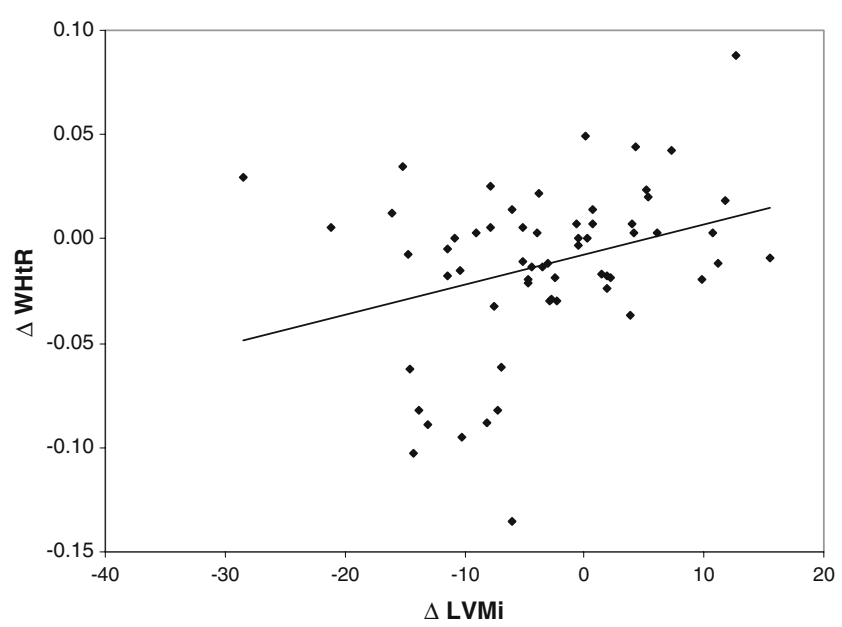

Fig. 2 Linear correlation between decrease in waist-to-height ratio $(W H t R)$ and decrease in left ventricular mass (LVMi): $r=0.263, p=0.03$ )

some authors did not find a significant difference between office BP and ABPM in predicting risk of left ventricular hypertrophy at the initial visit [22]. We performed ABPM in all children both at the start and after 12 months of treatment. Moreover, the decisions to intensify treatment were based on ABPM results after 3 and 6 months. Thus, we classified the severity of hypertension according to recently proposed criteria based on SBP values and SBP loads [9].

The clinical characteristics of our patients in terms of intermediate phenotype, metabolic abnormalities, and the extent of TOD were typical for children with PH [23, 24]. Subclinical cardiovascular injury and left ventricular hypertrophy are observed in $30-40 \%$ of children with PH already at diagnosis of elevated blood pressure [1-3, 23-25]. We used two cut-offs for left ventricular hypertrophy, i.e. for

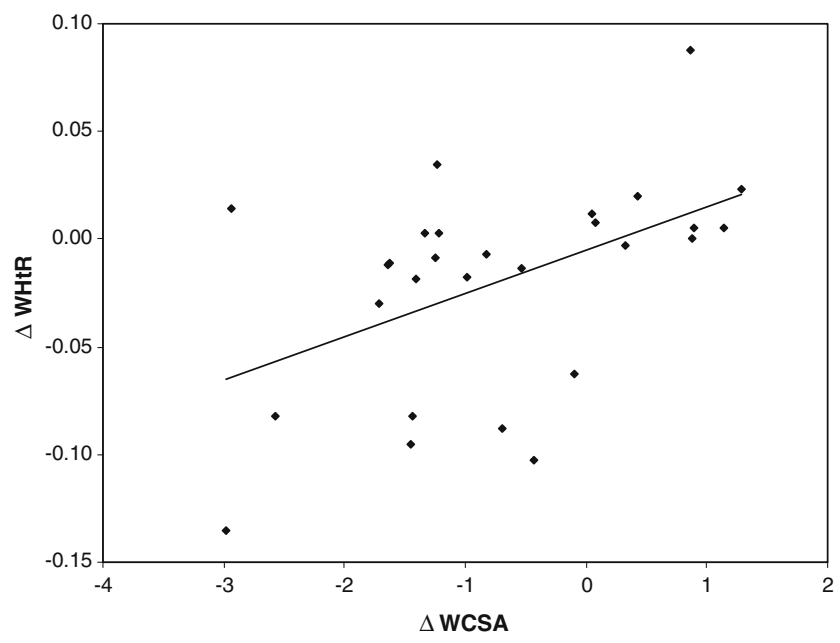

Fig. 3 Linear regression of correlation between the change in the WHtR and the change in wall cross-sectional area $(W C S A): r=0.528$, $p=0.002$
Table 4 Coefficients of Spearman's rank correlation test between pairs of variables describing regression of target organ damage and independent variables

\begin{tabular}{lll}
\hline & $r$ & $p$ \\
\hline$\Delta$ LVMi vs $\Delta$ WHtR & 0.263 & 0.03 \\
$\Delta$ LVMi vs $\Delta$ waist circumference & 0.318 & 0.01 \\
$\Delta$ cIMT-SDS vs $\Delta$ hsCRP & 0.332 & 0.01 \\
$\Delta$ WCSA-SDS vs $\Delta$ waist circumference & 0.490 & 0.006 \\
$\Delta$ WCSA-SDS vs $\Delta$ WHtR & 0.486 & 0.007 \\
$\Delta$ WCSA-SDS vs $\Delta$ WHR & 0.521 & 0.003 \\
$\Delta$ WCSA vs $\Delta$ WHtR & 0.528 & 0.002 \\
$\Delta$ WCSA vs $\Delta$ WHR & 0.528 & 0.002 \\
$\Delta$ WCSA vs $\Delta$ waist circumference & 0.573 & 0.001
\end{tabular}

LVMi left ventricular mass index; WHTR waist to height ratio; hsCRP high sensitivity $\mathrm{C}$ reactive protein; WCSA carotid wall cross sectional area; WHR waist to hip ratio

LVMi above the 95th percentile. One was based on the standardization of LVM to height and recommended by the 4th Task Report and by the European Society of Hypertension $[7,26]$, and the second was based on the standardization of LVM to height, age, and gender, and was proposed recently by Khoury et al. [15]. However, we did not find significant differences in the prevalence of left ventricular hypertrophy when using the two methods. It is because the age range of our patients was rather narrow and significant differences between the two methods of indexation of LVM are evident at younger and older ages.

We found that standard antihypertensive treatment based on lifestyle changes and/or blockade of the renin-angiotensin system is effective. Because BP, and particularly pulse pressure, is the main determinant of IMT, one can assume that an increased IMT is an indirect marker of long-lasting BP elevation and by causing increased stiffness may lead to resistance to treatment [27]. It corresponds with greater basal inflammatory activity shown as greater basal concentrations of hsCRP in non-responders as well as significant decreases in insulin resistance among responders. Moreover, a decrease in inflammatory activity expressed as a decrease in hsCRP concentration predicted a decrease in carotid IMT. In another study it was shown that innate immunity is activated in children with $\mathrm{PH}$ and that there is a significant relationship between markers of immune activity and TOD, between metabolic abnormalities and the amount of visceral fat [28]. These findings suggest that markers of inflammatory activity, insulin resistance, hypertensive arteriopathy, i.e. IMT, and of visceral obesity should be included as criteria for early and more intensive treatment in hypertensive children.

In adults, the extent of TOD correlates with BP and the lowering of BP leads to regression of TOD. It was also 
documented in cross-sectional studies that in children with $\mathrm{PH}$, the LVH correlates with hypertension severity [19-21]. In contrast, we did not find any direct relation between the hypotensive effect and regression of TOD. However, pediatric criteria for the diagnosis of hypertension are based on percentiles rather than absolute values. The measured absolute values of BP were numerically much lower than in adults with $\mathrm{PH}$ and on average did not even fulfill criteria for the diagnosis of hypertension in adults. Second, BP lowered by an average of $4 \mathrm{mmHg}$ and this may be too small a decrement to document the relation between the hypotensive effect and regression of TOD. On the other hand, BP measured both in the office and as ABPM physiologically rises with increasing age and height by approximately $1-2.5 \mathrm{mmHg}$ per year in adolescents. Therefore, the observed absolute average decrease of $4 \mathrm{mmHg}$ over 1 year of therapy is in fact even greater, because after 12 months our patients were both older and taller on average by $3 \mathrm{~cm}$, and their 95 th percentile values for BP (i.e. the upper limit of normal values) also increased, especially in adolescent boys.

Target organ damage in adolescents with $\mathrm{PH}$ is strictly related to metabolic abnormalities, central fat accumulation, and MS [18]. In the present study, the extent of metabolic abnormalities, including the prevalence of MS, was similar to that of our previous study [18]. We found that the prevalence of MS decreased in parallel to the regression of TOD. In addition, TOD regression was determined by a redistribution of adipose tissue, a decrease in central fat mass, and an increase in insulin sensitivity. This confirms the previous findings and also the hypothesis that metabolic abnormalities are the main determinants of TOD in adolescents with PH. It also indicates that the treatment of adolescents with $\mathrm{PH}$ should be complex, including the monitoring of anthropometrical parameters. A lack of significant changes in the BMI with a significant decrease in the WHR and WC indicate an increase in lean body mass, probably due to increased physical activity secondary to the adoption of lifestyle changes. In contrast, BMI, WHR, and WC did not change in patients who showed no regression of the TOD.

The potential effect of the pharmacological treatment on the redistribution of the fat tissue is another important issue. A prospective study showed that treatment with ACEi increases insulin sensitivity in adults [29]. There are also data indicating that ARBs increase insulin sensitivity by activation of PPAR $\gamma$ [30]. Because fat is a metabolically active tissue and its distribution is under hormonal control, it may well be that various pharmacological agents have a variable influence on the amount and distribution of fat tissue. It was recently shown that treatment with candesartan had no effect on insulin sensitivity and fat tissue distribution, but treatment with thiazides resulted in increased insulin resistance and an increase in visceral fat [31]. Our patients were instructed to increase their physical activity and all received dietary advice during each clinic visit. Thus, we were not able to separate the effects of pharmacological therapy from non-pharmacological therapy.

The main limitation of our study is its observational design. Therefore, the effects of non-pharmacological and pharmacological therapy could not be separated. Similarly, the effects of different antihypertensive drugs are difficult to compare. Second, because the adoption of lifestyle modifications includes both diet and physical activity, it is impossible to analyze the effects of each intervention separately. The other potential bias is compliance with treatment. Although we monitored patients every 3 months we were not able to fully eliminate drug non-compliance in those patients who did not lower BP.

Another important issue is the potential effect of the regression to the mean phenomenon. However, in all patients elevated BP was detected on many occasions before referral to our center and finally, diagnosis of $\mathrm{PH}$ was confirmed by ABPM. Moreover, we used ABPM both to classify the severity of hypertension and to monitor the effectiveness of the treatment. There is also the possibility that regression to the mean phenomenon may play a role in the observed decrease in LVMi and IMT. In contrast to BP measurements, results of both ECHO and ultrasound measurements are mainly dependent on investigator skills. Because changes in LVMi and IMT correlated with other investigator-independent variables, such as anthropometrical parameters, inflammatory activity, and insulin sensitivity markers, it seems less probable that the regression to the mean phenomenon played a significant role in the detection of changes in LVMi and IMT.

Because most adolescents with PH present with absolute $\mathrm{BP}$ values that could be classified as falling within the prehypertensive range for adults, it is intriguing that a good response to treatment in terms of BP lowering, regression of TOD, and a reduction in metabolic abnormalities might enable discontinuation of pharmacological treatment and continuation of only lifestyle modifications. Such an approach was tested in the TROPHY study with prehypertensive, middle-aged adults [32]. It seems logical that earlier treatment in a high-risk group of adolescents at an early stage of cardiovascular disease would have a more pronounced and long-lasting effect than in adults who are already hypertensive and one to two decades older.

\section{Conclusions}

Our results indicate that standard therapy of children with PH leads to significant BP lowering, regression of TOD and normalization of metabolic abnormalities. Decrease in 
blood pressure was associated with lower IMT, inflammatory activity, and increased insulin sensitivity. However, the main determinant of TOD regression was not blood pressure lowering by itself, but redistribution of the fat mass with a decrease in abdominal obesity. The positive effect of changes in body composition with an increase in lean body mass indicates that intensive non-pharmacological treatment might be helpful in reversing TOD.

Acknowledgement The authors would like to thank Malgorzata Syczewska, PhD, for help and consultation with statistical analysis.

Source of funding: the study was funded by grants from the Ministry of Science and Higher Education: 4253/BP01/2007/33 and 2268/BP01/2008/35.

Open Access This article is distributed under the terms of the Creative Commons Attribution Noncommercial License which permits any noncommercial use, distribution, and reproduction in any medium, provided the original author(s) and source are credited.

\section{References}

1. Daniels SR, Loggie JM, Khoury P, Kimball TR (1998) Left ventricular geometry and severe left ventricular hypertrophy in children and adolescents with essential hypertension. Circulation 97:1907-1911

2. Sorof J, Alexandrov AV, Garami Z, Turner JL, Grafe RE, Lai DL (2003) Carotid ultrasonography for detection of vascular abnormalities in hypertensive children. Pediatr Nephrol 18:1020-1024

3. Hanevold C, Waller J, Daniels S, Portman R, Sorof J, International Pediatric Hypertension Association (2004) The effects of obesity, gender and ethnic group on left ventricular hypertrophy and geometry in hypertensive children: a collaborative study of the International Pediatric Hypertension Association. Pediatrics 113:328-333

4. Bots ML, Dijk JM, Oren A, Grobbee E (2002) Carotid intimamedia thickness, arterial stiffness and risk of cardiovascular disease: current evidence. J Hypertens 20:2317-2325

5. Litwin M, Trelewicz J, Wawer ZT, Antoniewicz J, Wierzbicka A, Grenda R (2004) Intima-media thickness and functional properties of arterial wall in elastic and muscular type arteries in children and adolescents with arterial hypertension: controlled study. Pediatr Nephrol 19:767-774

6. Seeman T, Gilik J, Vondrak K, Simkova E, Flogelova M, Janda J (2007) Regression of left ventricular hypertrophy in children and adolescents with hypertension during ramipril monotherapy. Am J Hypertens 20:990-996

7. National High Blood Pressure Education Program Working Group on High Blood Pressure in Children and Adolescents (2004) The fourth report on diagnosis, evaluation and treatment of high blood pressure in children and adolescents. Pediatrics 114:555-576

8. Wuhl E, Witte K, Soergel M, Mehls O, Shaeffer F, German Working Group on Pediatric Hypertension (2002) Distribution of 24-h ambulatory blood pressure in children: normalized reference values and role of body dimensions. J Hypertens 20:1995-2007

9. Urbina E, Alpert B, Flynn J, Hayman L, Harshfield GA, Jacobson M, Mahoney L, McCrindle B, Mietus-Snyder M, Steinberger J, Daniels S (2008) Ambulatory blood pressure monitoring in children and adolescents: recommendations for standard assessment: a scientific statement from the American Heart Association Atherosclerosis, Hypertension and Obesity in Youth Committee of the Council on
Cardiovascular Disease in the Young and the Council for High Blood Pressure Research. Hypertension 52:433-451

10. Cole TJ, Bellizzi MC, Flegal KM, Dietz WH (2000) Establishing a standard definition for child overweight and obesity worldwide: international study. BMJ 320:1240-1243

11. Jourdan C, Wuehl E, Litwin M, Fahr K, Trelewicz J, Jobs K, Schenk JP, Grenda R, Mehls O, Troeger J, Schaefer F (2005) Normative values of intima-media thickness and distensibility of large arteries in healthy adolescents. J Hypertens 23:1707-1715

12. Daniels SR, Kimball TR, Morrison JA, Khoury P, Meyer RA (1995) Indexing left ventricular mass to account for differences In body size in children and adolescents without cardiovascular disease. Am J Cardiol 76:699-701

13. De Simone G, Deveraux RB, Daniels SR, Koren MJ, Meyer RA, Laragh JH (1995) Effect of growth on variability of left ventricular mass: assessment of allometric signals in adults and children and their capacity to predict cardiovascular risk. J Am Coll Cardiol 25:1056-1062

14. Daniels SR, Meyer RA, Liang Y, Bove KE (1998) Echocardiographically determined left ventricular mass index in normal children, adolescents and young adults. J Am Coll Cardiol 12:703-708

15. Khoury PR, Mitsnefes MS, Daniels SR, Kimball TR (2009) Age specific reference intervals for indexed left ventricular mass index in children. J Am Soc Echocardiogr 22:709-714

16. Gutt M, Davis CL, Spitzer SB, Llabre MM, Kumar M, Czarnecki EM, Schneiderman N, Skyler JS, Marks JB (2000) Validation of the insulin sensitivity index (ISI $(0,120))$ : comparison with other measures. Diabetes Res Clin Pract 47:1777-1784

17. Schwartz GJ, Brion LP, Spitzer A (1987) The use of plasma creatinine concentration for estimating glomerular filtration rate in infants, children and adolescents. Pediatr Clin North Am 34:571-590

18. Litwin M, Sladowska J, Antoniewicz J, Niemirska A, Wierzbicka A, Daszkowska J, Wawer ZT, Janas R, Grenda R (2007) Metabolic abnormalities, insulin resistance and metabolic syndrome in children with primary hypertension. Am J Hypertens $20: 875-882$

19. Sorof JM, Cardwell G, Franco K, Portman RJ (2002) Ambulatory blood pressure and left ventricular mass in hypertensive children. Hypertension 39:903-908

20. Richey PA, Disessa TG, Hastings MC, Somes GW, Akpert BS, Jones DP (2008) Ambulatory blood pressure and increased left ventricular mass in children at risk for hypertension. J Pediatr 152:343-348

21. McNiece KL, Gupta-Malhorta M, Samuels J, Bell C, Garcia K, Pottenberger T, Sorof JM, Portman RJ (2007) Left ventricular hypertrophy in hypertensive adolescents. Analysis of risk by 2004 National High Blood Pressure Education Program Working Group criteria. Hypertension 50:392-395

22. Brady TM, Fivush B, Flynn JT, Parekh R (2008) Ability of blood pressure to predict left ventricular hypertrophy in children with primary hypertension. J Pediatr 152:73-78

23. Flynn JT, Alderman MH (2005) Characteristics of children with primary hypertension seen at a referral center. Pediatr Nephrol 20:961-966

24. Litwin M, Niemirska A, Sladowska J, Antoniewicz J, Daszkowska J, Wierzbicka A, Wawer ZT, Grenda R (2006) Left ventricular hypertrophy and arterial wall thickening in children with essential hypertension. Pediatr Nephrol 21:811-819

25. Lande MB, Carson NL, Roy J, Meagher CC (2006) Effects of childhood primary hypertension on carotid intima media thickness: a matched controlled study. Hypertension 48:40-44

26. Lurbe E, Cifkova R, Cruickshank JK, Dillon MJ, Ferreira I, Invitti C, Kuznetsova T, Laurent S, Mancia G, Morales-Olivas F, Rascher W, Redon J, Schaefer F, Seeman T, Stergiou G, Wuhl E, Zanchetti A (2009) Management of high blood pressure in 
children and adolescents: recommendations of the European Society of Hypertension. J Hypertens 27:1719-1742

27. Laurent S (1995) Arterial wall hypertrophy and stiffness in essential hypertensive patients. Hypertension 26:355-362

28. Litwin M, Michalkiewicz J, Niemirska A, Gockowska L, Kubiszewska I, Wierzbicka A, Wawer ZT, Janas R (2010) Inflammatory activity in children with primary hypertension. Pediatr Nephrol 25:1711-1718

29. Black HR, Davis B, Barzilay J (2008) Metabolic and clinical outcomes in nondiabetic individuals with the metabolic syndrome assigned to chlorthalidone, amlodipine, or lisinopril as initial treatment for hypertension: a report from the Antihypertensive and Lipid-Lowering Treatment to Prevent Heart Attack Trial (ALLHAT). Diabetes Care 31:353-360
30. Benson SC, Pershadsingh HA, Ho CI (2004) Identification of telmisartan as a unique angiotensin II receptor antagonist with selective PPAR $\gamma$-modulating activity. Hypertension 43:9931002

31. Eriksson JW, Jansson PA, Carlberg B (2008) Hydrochlorothiazide, but not candesartan, aggravates insulin resistance and causes visceral and hepatic fat accumulation: the mechanisms for the diabetes preventing effect of candesartan (MEDICA) study. Hypertension 52:1030-1037

32. Julius S, Nesbitt SD, Egan BM, Weber BM, Michelson EL, Kacirotti N, Black HR, Grimm RH Jr, Messerli FH, Oparil S, Schork MA, Trial of Preventing Hypertension (Trophy) Study investigators (2006) Feasibility of treating prehypertension with an angiotensin receptor blocker. N Eng J Med 354:1685-1697 